\title{
Excellent Functional Outcomes and Low Complication Rates Following Knotless Arthroscopic Bankart Repair: A Systematic Review of Clinical and Biomechanical Studies
}

\author{
Xin Y. Mei, M.D., Ujash Sheth, M.D., M.S.c., F.R.C.S.C., and Jihad Abouali, M.D., F.R.C.S.C.
}

Purpose: To provide a comprehensive review of the current clinical and biomechanical evidence for the use of knotless suture anchors during arthroscopic Bankart repair. Methods: A comprehensive search of 5 electronic databases from inception to July 2020 was performed for clinical and biomechanical studies in English evaluating outcomes following arthroscopic Bankart repair using knotless suture anchors. Two independent reviewers assessed articles for inclusion. Risk of bias was assessed using the Methodological Index for Non-Randomized Studies (MINORs) criteria. Clinical outcomes of interest and key findings from biomechanical studies were summarized. Results: Ten clinical studies (430 knotless repairs), including 4 cohort studies directly comparing knotless $(\mathrm{N}=117)$ to knot-tying repairs $(\mathrm{N}=192)$, were deemed eligible. Four biomechanical studies were also identified. Heterogeneity and the lack of randomized studies precluded data pooling and quantitative meta-analysis. Rates of redislocation ranged from $2.2 \%$ to $14.7 \%$ and $1.5 \%$ to $23.8 \%$ for knottying and knotless repair, respectively. Both knot-tying and knotless repair demonstrated excellent postoperative functional outcome, as assessed by visual analog scale, Constant-Murley, Rowe, and QuickDASH scores, with $80 \%$ to $94 \%$ of patients returning to sports participation. Biomechanical studies showed similar stiffness and load to failure between knottying and knotless repairs, with the most common mode of failure being retear at the suture-soft tissue interface. Conclusions: Current level II to IV clinical and biomechanical evidence supports knotless arthroscopic Bankart repair as an effective procedure with excellent functional outcomes and low rates of complication and reoperation. Level of Evidence: Level IV, systematic review of level II to IV studies.

A rthroscopic Bankart repair using suture anchors is a widely accepted method of restoring labral anatomy, with results similar to that of open stabilization. ${ }^{1,2}$ The benefits of arthroscopic surgery include faster return to activity, smaller surgical scars, improved range of motion, and shorter hospital stay. ${ }^{3}$

From the Division of Orthopaedic Surgery, Michael Garron Hospital, Toronto, Ontario, Canada (X.Y.M., J.A.) and Division of Orthopaedic Surgery, Sunnybrook Health Sciences Centre, Toronto, Ontario, Canada.

The authors report that they have no conflicts of interest in the authorship and publication of this article. Full ICMJE author disclosure forms are available for this article online, as supplementary material.

Received August 24, 2020; accepted January 24, 2021.

Address correspondence to Xin Y. Mei, M.D., Division of Orthopaedic Surgery, Michael Garron Hospital, 825 Coxwell Ave, Suite 304, Toronto, ON, M4G 5T2, Canada.E-mail:xmei@qmed.ca

(C) 2021 THE AUTHORS. Published by Elsevier Inc. on behalf of the Arthroscopy Association of North America. This is an open access article under the CC BY-NC-ND license (http://creativecommons.org/licenses/by-nc-nd/4.0/). 2666-061X/201432

https://doi.org/10.1016/j.asmr.2021.01.014
Two techniques of capsulolabral repair with different suture anchor designs have been described in the literature: a traditional arthroscopic knot-tying technique and a knotless technique. While both are widely used, knotless suture anchors are becoming increasingly popular due to their potential to mitigate complications associated with arthroscopic knot tying such as knot migration and soft tissue irritation from a highprofile knot. Another proposed advantage of knotless repair is improved efficiency and reproducibility secondary to avoiding the technical challenge of arthroscopic knot tying and subsequent improved workflow. ${ }^{4}$

The current available clinical outcome data on knotless arthroscopic Bankart repair are limited to small, singlecenter series. Furthermore, there remains a lack of consensus and clinical equipoise regarding the use of traditional knot-tying vs knotless techniques. The purpose of this study was to provide a comprehensive review of the current clinical and biomechanical evidence for the use of knotless suture anchors during arthroscopic Bankart repair. We hypothesized that arthroscopic 
Bankart repair using knotless suture anchors will provide excellent functional outcomes and low rates of complication and reoperation.

\section{Methods}

\section{Literature Search}

A systematic review was conducted according to the Preferred Reporting Items for Systematic Reviews and Meta-Analyses guidelines. ${ }^{5}$ Studies were identified using the following electronic databases: MEDLINE, MEDLINE In-Process, EMBASE, Cochrane Database of Systematic Reviews, and Cochrane Controlled Trials Register. The databases were searched from inception to July 2020 using the following MeSH headings and text keywords: shoulder, suture, knots, anchors, knotless, bioknotless, and traditional knot. The full electronic search strategy is presented in the Appendix. The cited literature in the included studies was also reviewed to identify any additional relevant articles not found in our initial literature search.

\section{Inclusion and Exclusion Criteria}

All studies reporting clinical outcomes among patients aged 16 years and older following knotless arthroscopic Bankart repair with a minimum 2-year follow-up were included. Given the relative novelty of knotless suture anchors and the scarcity of level I evidence available, we chose to include nonrandomized studies. In addition, biomechanical studies comparing knot-tying and knotless Bankart repair were included. Studies were excluded if they evaluated patients with bony Bankart lesions, significant glenoid or humeral head bone loss, SLAP tears, humeral avulsion of glenohumeral ligament lesions, open Bankart repairs, or revision procedures. Review articles, technical descriptions, studies with less than 2 years of clinical follow-up, and studies with a sample size $<10$ patients were also excluded. A full description of inclusion and exclusion criteria is presented in Table 1.

Clinical outcomes of interest were visual analog scale (VAS) pain score, Constant-Murley score, Rowe shoulder score, QuickDASH score, and rates of recurrent instability, anchor pull-out, reoperation, and return to sport percentage.

\section{Study Selection and Data Abstraction}

All abstracts and titles were reviewed independently in an unbiased standardized manner by 2 authors (X.Y.M. and U.S.). The abstracts were sorted by predefined inclusion criteria and classified as relevant, possibly relevant, or irrelevant. Full-text review of the "possibly relevant" studies was done for further clarification. Disagreement between reviewers was resolved through discussion and consensus with the senior author (J.A.).

The following data were extracted from each clinical study meeting the inclusion criteria: study design, patient sample and characteristics (including mean age, male to female ratio), preoperative differences between cohorts, outcomes studied, and measures of association between intervention and various outcomes, including $P$ values. Data extracted from biomechanical studies include study design, implants used, construct stiffness, load to failure, failure mode, and main study conclusions.

Table 1. Inclusion and Exclusion Criteria

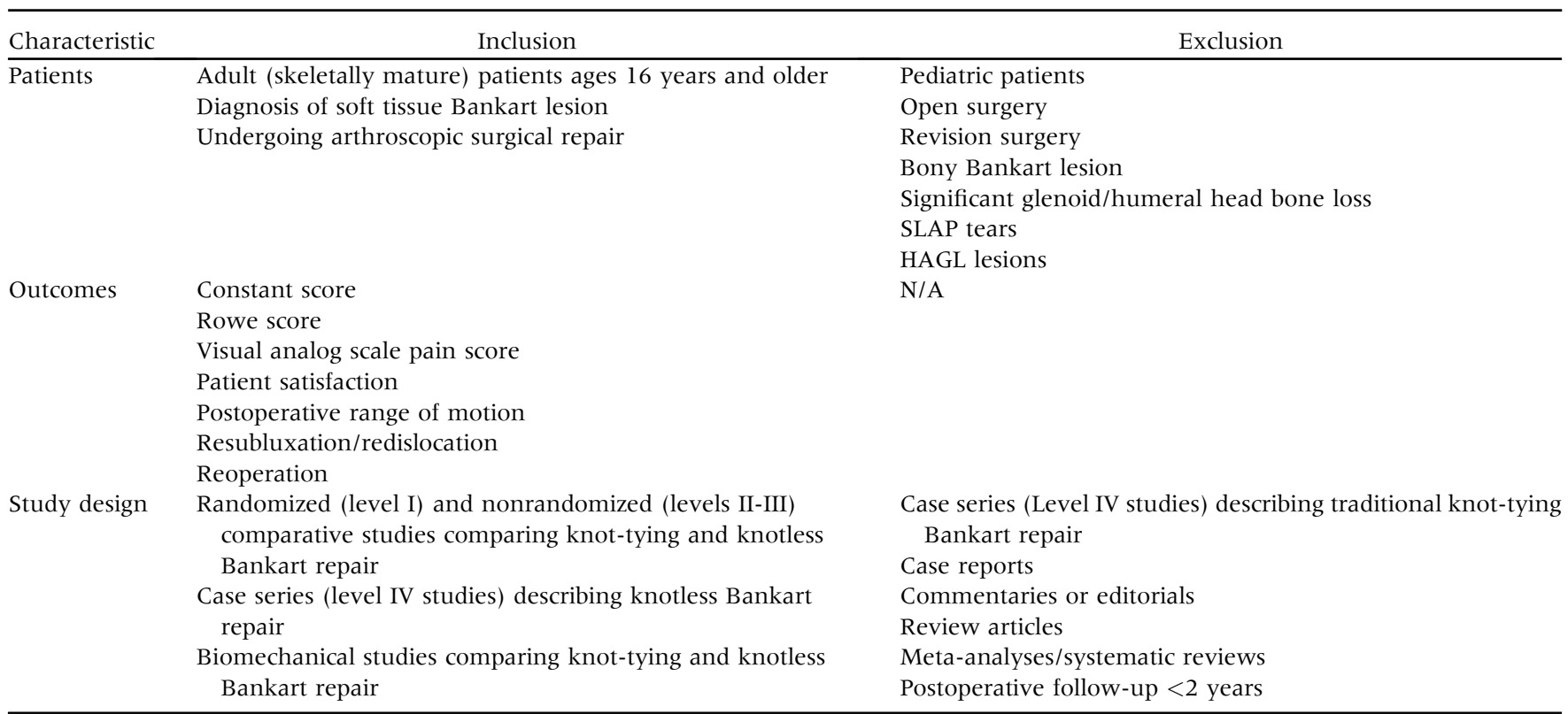

HAGL, humeral avulsion of glenohumeral ligament; N/A, not applicable. 


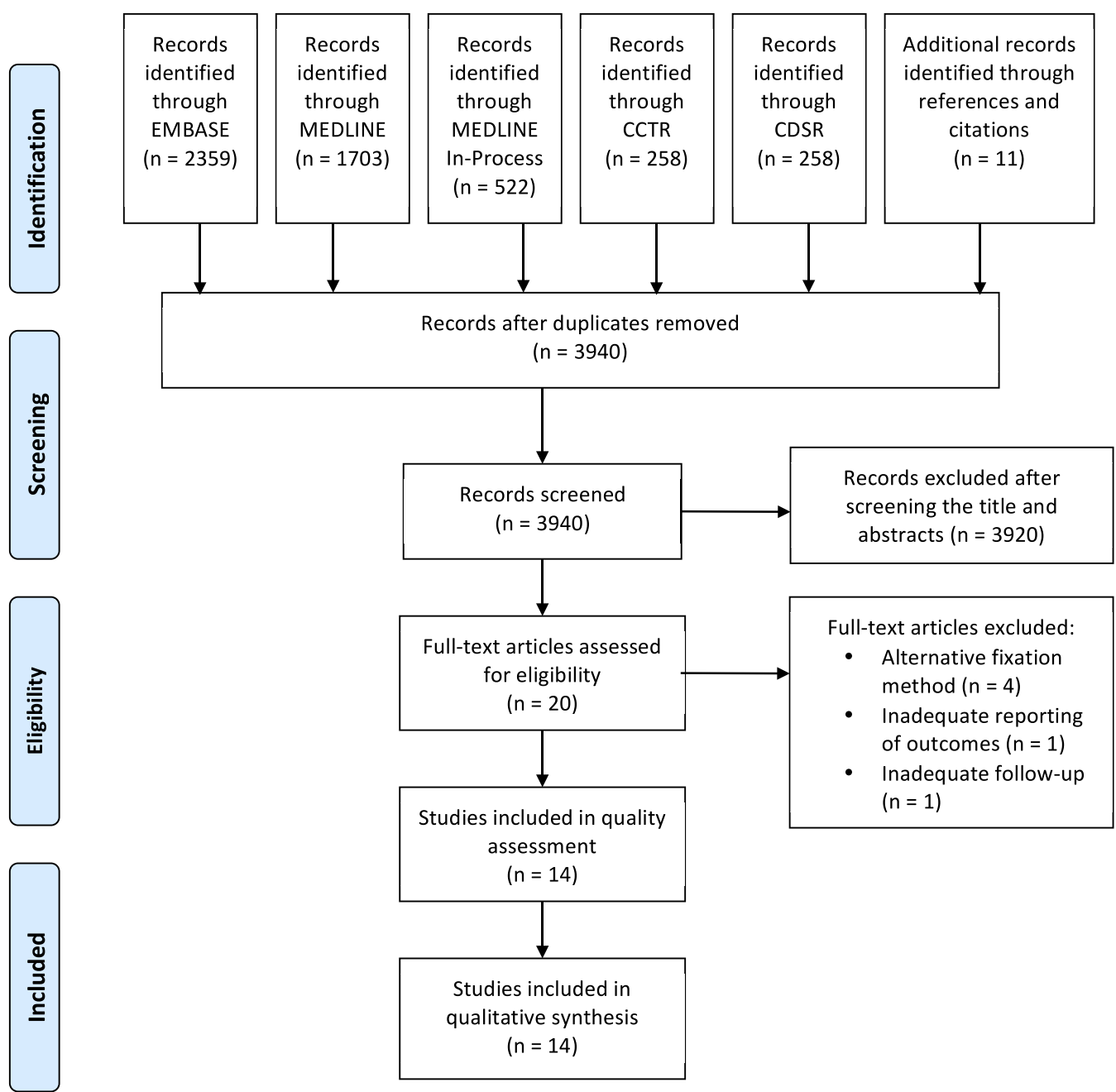

Fig 1. Flow diagram demonstrating the selection of studies for the systematic review.

\section{Methodological Quality Assessment and Risk of Bias}

Two authors (X.Y.M. and U.S.) independently rated the methodological quality of each included clinical study using the Methodological Index for NonRandomized Studies (MINORS) criteria, ${ }^{6}$ which is a validated 8 - or 12-item checklist designed to analyze the quality of noncomparative and comparative, nonrandomized, surgical studies. Each checklist item is scored from 0 to 2 based on whether it is not addressed, partially addressed, or completed addressed by the study in question.

\section{Statistical Analysis}

Descriptive statistics were used to summarize patient demographic characteristics and outcome measures. Forest plots were constructed to visualize outcomes of interest across studies. Data heterogeneity was quantified using the $I^{2}$ statistic. An $I^{2}$ value of $0 \%$ represents no heterogeneity, and values of $25 \%, 50 \%$, and $\geq 75 \%$ represent low, moderate, and high levels of heterogeneity, respectively. ${ }^{7}$ All statistical analyses were performed using Cochrane Review Manager Version 5.3 (Cochrane Collaboration).

\section{Results}

\section{Study Characteristics}

The initial search yielded 3940 citations following the exclusion of duplicates. Ten clinical studies and 4 biomechanical studies were deemed eligible following this rigorous review process. The process of study selection is included in Fig 1.

Four cohort studies directly comparing knot-tying and knotless Bankart repair and 6 case series evaluating knotless repair met inclusion criteria. A total of 192 knottying and 430 knotless arthroscopic Bankart repairs were included. Surgical date ranged from 1999 to 2011 , and sample size ranged from 20 to 102 patients. Mean reported age ranged from 22 to 26.7 years, with $85.5 \%$ (532/622) male patients. Mean duration of follow-up ranged from 2.3 to 8 years. Knot configurations used in knot-tying repair included horizontal mattress, ${ }^{8}$ sliding hangman's knot with alternating half-stitches, ${ }^{9,10}$ and 
Table 2. Demographic and Outcome Data of the Included Clinical Studies

\begin{tabular}{|c|c|c|c|c|c|c|c|c|c|c|c|c|c|}
\hline Study (Year) & $\begin{array}{l}\text { Study Design } \\
\text { (Level } \\
\text { of Evidence) }\end{array}$ & $\begin{array}{l}\text { Date of } \\
\text { Surgery }\end{array}$ & $\begin{array}{l}\text { KT, } \\
\text { No. }\end{array}$ & $\begin{array}{l}\text { KL, } \\
\text { No. }\end{array}$ & $M: F$ & $\begin{array}{l}\text { Age, Mean } \\
\text { (Range), y }\end{array}$ & $\begin{array}{c}\text { Follow-up, Mean } \\
\text { (Range) }\end{array}$ & $\begin{array}{l}\text { Mean } \\
\text { Constant } \\
\text { Score }\end{array}$ & $\begin{array}{l}\text { Mean } \\
\text { Rowe } \\
\text { Score }\end{array}$ & $\begin{array}{c}\text { Mean VAS } \\
\text { (Maximum 10) }\end{array}$ & $\begin{array}{c}\text { Recurrent } \\
\text { Instability (\%) }\end{array}$ & $\begin{array}{c}\text { Anchor } \\
\text { Pullout } \\
(\%)\end{array}$ & $\begin{array}{c}\text { Reoperation } \\
\text { for Instability } \\
(\%)\end{array}$ \\
\hline $\begin{array}{c}\mathrm{Ng} \mathrm{et} \mathrm{al.} \\
(2014)\end{array}$ & $\begin{array}{l}\text { Prospective } \\
\text { cohort (II) }\end{array}$ & $2007-2011$ & 45 & 42 & $77: 11$ & $21(17-31)$ & 2.7 y $(2.0-3.7$ y) & $\begin{array}{c}\text { KT: } 64 \text { to } 92 \\
(P=.024) \\
\text { KL: } 62 \text { to } 89 \\
(P=.019)\end{array}$ & NR & $\begin{array}{c}\text { KT: } 2.5 \text { to } 0.7 \\
(P=.017) \\
\text { KL: } 2.8 \text { to } 0.9 \\
(P=.011)\end{array}$ & $\begin{array}{ll}\text { KT: } 1 & (2.2 \%) \\
\text { KL: } 1 & (2.4 \%)\end{array}$ & None & $\begin{array}{l}\text { KT: } 1(2.2 \%) \\
\text { KL: } 1(2.4 \%)\end{array}$ \\
\hline $\begin{array}{l}\text { Kocaoglu } \\
\text { et al. }^{9}(2009)\end{array}$ & $\begin{array}{l}\text { Prospective } \\
\text { cohort (II) }\end{array}$ & 2004-2006 & 18 & 20 & $26: 12$ & $23(17-32)$ & $40 \mathrm{mo}(26-56 \mathrm{mo})$ & $\mathrm{NR}$ & $\begin{array}{c}\mathrm{KT}: 41 \text { to } 92 \\
(P=.0032) \\
\mathrm{KL}: 43 \text { to } 91 \\
(P=.0038)\end{array}$ & $\mathrm{NR}$ & $\begin{array}{ll}\text { KT: } 1 & (5.6 \%) \\
\text { KL: } 1 & (8.3 \%)\end{array}$ & None & $\begin{array}{l}\text { KT: } 1(5.6 \%) \\
\text { KL: } 1(8.3 \%)\end{array}$ \\
\hline $\begin{array}{l}\text { Wu et al. } \\
\quad(2020)\end{array}$ & $\begin{array}{l}\text { Retrospective } \\
\text { cohort (III) }\end{array}$ & NR & 68 & 34 & $89: 13$ & 22.5 & $4.8 \pm 2.5 y$ & NR & $\begin{array}{c}\text { KT: } 92.2 \\
\text { postoperatively } \\
\text { KL: } 90.5 \\
\text { postoperatively }\end{array}$ & $\begin{array}{c}\text { KT: } 0.8 \text { post } \\
\text { operatively } \\
\text { KL: } 1.3 \\
\text { postoperatively }\end{array}$ & $\begin{array}{c}\text { KT: } 10(14.7 \%) \\
\text { KL: } 3(8.8 \%)\end{array}$ & None & $\begin{aligned} \text { KT: } 10 & (14.7 \%) \\
\text { KL: } 1 & (2.9 \%)\end{aligned}$ \\
\hline $\begin{array}{l}\text { Cho et al. }{ }^{11} \\
\text { (2006) }\end{array}$ & $\begin{array}{l}\text { Retrospective } \\
\text { cohort (III) }\end{array}$ & 2001-2002 & 61 & 21 & $75: 7$ & $24(16-42)$ & 29 mo (24-42 mo) & $\begin{array}{c}\text { KT: } 62 \text { to } 91 \\
(P<.001) \\
\text { KL: } 66 \text { to } 86 \\
(P<.001)\end{array}$ & $\begin{array}{c}\text { KT: } 45 \text { to } 93 \\
(P<.001) \\
\text { KL: } 42 \text { to } 86 \\
(P<.001)\end{array}$ & $\begin{array}{c}\text { KT: } 2.3 \text { to } 0.4 \\
(P<.01) \\
\text { KL: } 3.2 \text { to } 1.7 \\
\quad(\mathrm{NS})\end{array}$ & $\begin{array}{c}\text { KT: } 3(4.9 \%) \\
\text { KL: } 5(23.8 \%)\end{array}$ & None & $\begin{array}{c}\text { KT: } 1(1.6 \%) \\
\text { KL: } 4(19.0 \%)\end{array}$ \\
\hline $\begin{array}{l}\text { Alentorn-Geli } \\
\text { et al. }{ }^{14} \\
(2016)\end{array}$ & Case series (IV) & $2002-2009$ & - & 57 & $57: 0$ & $22(16-28)$ & 8 y $(5-10 y)$ & NR & $\begin{array}{c}80 \text { post } \\
\text { operatively }\end{array}$ & $\mathrm{NR}$ & $6(10.5 \%)$ & None & $4(7.0 \%)$ \\
\hline $\begin{array}{l}\text { Stein et al. }{ }^{12} \\
\quad(2011)\end{array}$ & Case series (IV) & $2006-2007$ & - & 47 & $39: 8$ & 26.9 & $32 \mathrm{mo}$ & $\begin{array}{l}67.1 \text { to } 94.0 \\
(P<.001)\end{array}$ & $\begin{array}{c}61.6 \text { to } 95.9 \\
(P<.001)\end{array}$ & NR & $5(10.6 \%)$ & None & $5(10.6 \%)$ \\
\hline $\begin{array}{l}\text { Park et al. }{ }^{16} \\
(2009)\end{array}$ & Case series (IV) & $2001-2005$ & - & 69 & $57: 12$ & $26(17-51)$ & $40 \mathrm{mo}(23-60 \mathrm{mo})$ & NR & $\begin{array}{c}43.1 \text { to } 93.8 \\
(P<.01)\end{array}$ & $\begin{array}{l}3.4 \text { to } 0.9 \\
(P<.01)\end{array}$ & $1(1.5 \%)$ & $2(2.9 \%)$ & $1(1.5 \%)$ \\
\hline $\begin{array}{l}\text { Thal et al. }{ }^{13} \\
\text { (2007) }\end{array}$ & Case series (IV) & $1998-2003$ & - & 73 & $57: 16$ & $26.7(16-64)$ & NR (2-7 y) & NR & $\begin{array}{l}41 \text { to } 93.4 \\
(P<.001)\end{array}$ & $\mathrm{NR}$ & $5(6.9 \%)$ & None & $4(5.5 \%)$ \\
\hline $\begin{array}{l}\text { Hayashida } \\
\text { et al. }{ }^{15} \\
(2006)\end{array}$ & Case series (IV) & $2000-2002$ & - & 47 & $38: 9$ & $26(16-49)$ & 28 mo $(24-38)$ & NR & $\begin{array}{l}31 \text { to } 91 \\
(P<.001)\end{array}$ & NR & $3(6.4 \%)$ & $1(2.1 \%)$ & $1(2.1 \%)$ \\
\hline $\begin{array}{l}\text { Garofalo } \\
\text { et al. }^{17} \\
(2005)\end{array}$ & Case series (IV) & $1999-2000$ & - & 20 & $17: 3$ & $23.2(24-34)$ & $43 \mathrm{mo}(36-48 \mathrm{mo})$ & $\begin{array}{c}92 \text { post } \\
\text { operatively }\end{array}$ & $\begin{array}{l}93 \text { post } \\
\text { operatively }\end{array}$ & $\begin{array}{c}3 \text { patients had } \\
1-2 / 10 \text { post } \\
\text { operatively }\end{array}$ & $1(5.0 \%)$ & None & None \\
\hline
\end{tabular}

KL, knotless Bankart repair; KT, knot-tying Bankart repair; NR, not reported; NS, not significant; VAS, visual analog scale. 


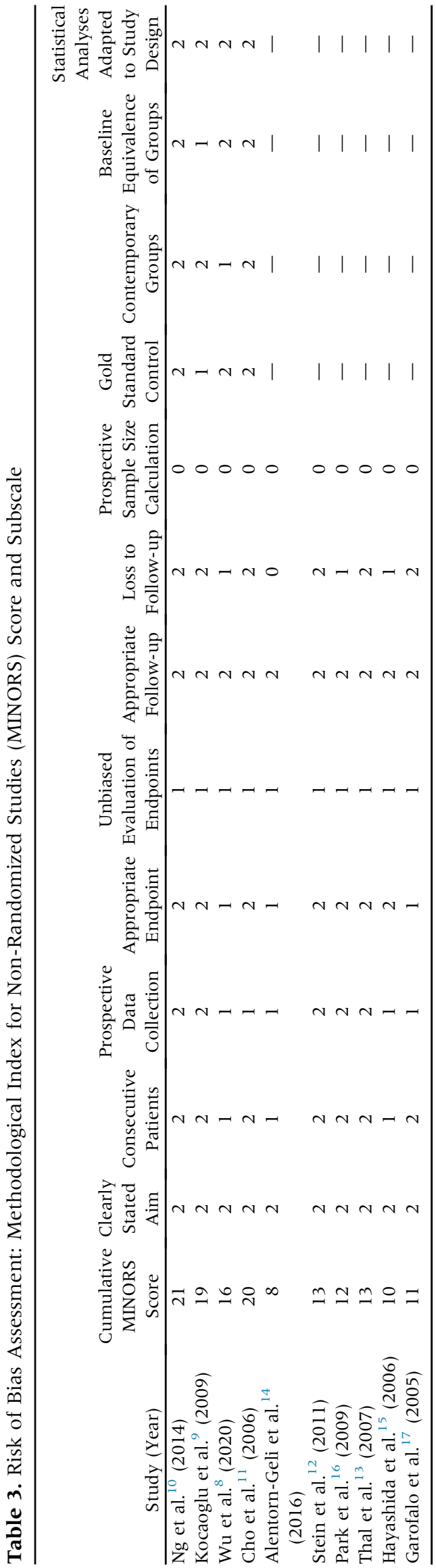

nonsliding Revo knot with alternating half-stitches. ${ }^{11}$ Most knotless anchors used are PEEK (polyether ether ketone) anchors manufactured by Arthrex (PushLock) ${ }^{8-10,12}$ or Depuy (Mitek Bioknotless). ${ }^{11,13,14}$ Three case series on knotless repair used metallic knotless anchors manufactured by Depuy. ${ }^{15-17}$ A summary of included clinical studies is presented in Table 2.

\section{Methodologic Quality Assessment and Risk of Bias}

Four cohort studies and 6 case series underwent methodologic quality assessment using MINORS criteria. This is summarized in Table 3. All studies clearly stated the primary aim and had appropriate length of follow-up. All comparative studies had contemporary control groups with equivalent baseline patient characteristics and performed appropriate statistical analyses. No studies prospectively calculated sample size or evaluated clinical endpoints in a blinded fashion. Approximately half the studies were retrospective in nature and had greater than 5\% loss to follow-up. The mean cumulative MINORS score was 19 of $24(16-21)$ for comparative studies and 11.2 of 16 (8-13) for case series. Data pooling and quantitative meta-analysis were not possible due to the low level of evidence and heterogeneity across studies.

\section{Recurrent Instability and Reoperation}

All 10 clinical studies, ${ }^{8-17}$ involving 192 knot-tying and 430 knotless repairs, reported rates of recurrent instability and reoperation. Forest plots summarizing consistently reported outcomes from individual studies with corresponding effect sizes are presented in Fig 2 and Fig 3. Rates of redislocation ranged from $2.2 \%$ to $14.7 \%$ and $1.5 \%$ to $23.8 \%$ for knot-tying and knotless repair, respectively. Rates of reoperation for instability ranged from $2.2 \%$ to $14.7 \%$ and $0 \%$ to $19.0 \%$ for knottying and knotless repair, respectively. There was moderate heterogeneity across studies for both recurrent instability $\left(I^{2}=42 \%\right)$ and reoperation for instability $\left(I^{2}=37 \%\right)$.

Two of the 4 cohort studies comparing knot-tying and knotless repair, ${ }^{9,10}$ reported no difference in the rate of redislocation between groups. Cho et al. ${ }^{11}$ reported significantly higher redislocation rates in their knotless group compared to their knot-tying group $(23.8 \%$ vs $4.9 \%, P=.012)$. Of note, their study cohort underwent surgery at an earlier time period (2001-2002) using a different design of knotless suture anchor in comparison to the other studies included in this review. Conversely, $\mathrm{Wu}$ et al. $^{8}$ reported significantly higher rates of resubluxation (28\% vs 9\%, $P=.039)$ but not redislocation $(15 \%$ vs $9 \%, P>.05)$ or reoperation (18\% vs $3 \%, P>.05)$ in the knot-tying group as compared with the knotless group.

Patient risk factors for recurrent instability such as glenoid bone loss and large Hill-Sachs lesions are not 


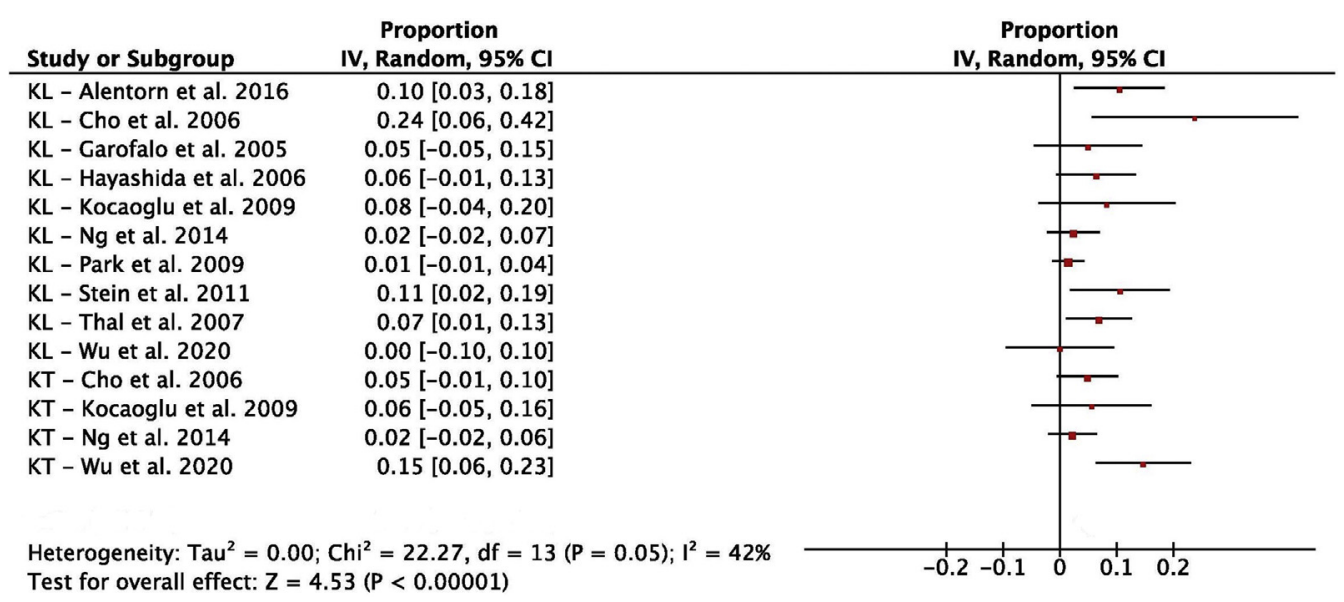

Fig 2. Forest plot demonstrating reported rates of redislocation across included studies. KL, knotless Bankart repair; KT, knot-tying Bankart repair. consistently reported by the included studies. Most studies considered glenoid bone loss $>20 \%$ to $25 \%$ and off-track engaging Hill-Sachs lesions to be contraindications to arthroscopic Bankart repair. The presence of Hill-Sachs lesions was reported by 5 studies and ranged from $59 \%$ to $100 \% .^{8,9,13,14,17}$ No knot-related complications such as soft tissue irritation or chondral abrasion have been reported by the included studies.

\section{Anchor Pullout}

Two studies involving an older-design, all-metal knotless suture anchor (Depuy Mitek) reported anchor pullout as a complication. Hayashida et al. ${ }^{15}$ reported 1 patient $(2.1 \%)$ with radiographically diagnosed anchor pullout 2 months postoperatively. There was no preceding trauma or dislocation, and the patient was asymptomatic with the exception of subjective shoulder stiffness. The patient underwent arthroscopic removal of the loose suture anchor. Intraoperative assessment revealed slight backing out of the anchor into the glenohumeral joint with subsequent humeral head cartilage damage by the tail of the anchor. The capsulolabral repair, anchor loop, and the anchor itself were found to be intact. The authors postulated inadequate depth of insertion and failure to stress the anchor to ensure secure fixation after insertion as possible causes of failure.

Similarly, Park et al. ${ }^{16}$ reported anchor pullout requiring arthroscopic removal in 2 patients $(2.9 \%)$. Intraoperative assessment during revision surgery revealed focal humeral head articular damage secondary to protruded anchor tails. Although not firmly fixed, the protruded anchors were not freely movable and not loose enough to be removed without effort. Furthermore, the authors found perianchor lucency on plain radiographs to be a risk factor for impending anchor pullout and redislocation.

\section{VAS}

Five studies, $8,10,11,16,17$ involving 174 knot-tying and 186 knotless repairs, reported either a significant improvement from preoperative to postoperative or excellent postoperative VAS pain scores. The mean VAS score ranged from 2.3 to 2.5 preoperatively and 0.4 to
Fig 3. Forest plot demonstrating reported rates of reoperation for instability across included studies. KL, knotless Bankart repair; KT, knot-tying Bankart repair.

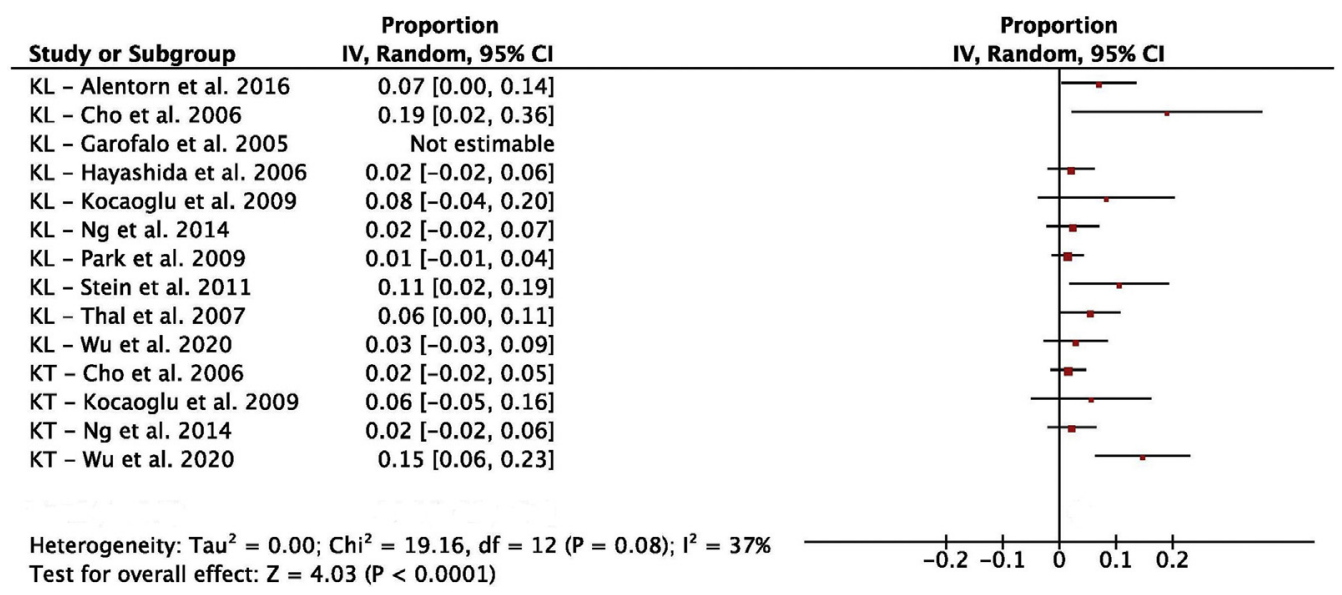


Table 4. Summary of the Included Biomechanical Studies

\begin{tabular}{|c|c|c|c|c|c|c|c|c|}
\hline Study (Year) & $\begin{array}{c}\text { No. of } \\
\text { Cadaver } \\
\text { Shoulders }\end{array}$ & Study Design & KT & KL & Stiffness $(\mathrm{N} / \mathrm{mm})$ & Load to Failure $(\mathrm{N})$ & Failure Mode & Conclusions \\
\hline $\begin{array}{c}\text { Lacheta et al. }{ }^{4} \\
(2020)\end{array}$ & 30 & $\begin{array}{l}12 \text { pairs of shoulders } \\
\text { randomized to } \\
\text { knot-tying vs } \\
\text { knotless repair } \\
\text { (contralateral } \\
\text { side): half simple } \\
\text { stitch, half } \\
\text { horizontal } \\
\text { mattress stitch } \\
\text { Remaining } 6 \\
\text { shoulders left } \\
\text { intact to test } \\
\text { strength of native } \\
\text { labrum }\end{array}$ & $\begin{array}{l}\text { Two knotted } \\
\text { 1.8-mm } \\
\text { FiberTak } \\
\text { anchors } \\
\text { (Arthrex) } \\
\end{array}$ & $\begin{array}{l}\text { Three knotless } \\
\text { 1.8-mm } \\
\text { FiberTak } \\
\text { anchors } \\
\text { (Arthrex) }\end{array}$ & $\begin{array}{l}\text { KT simple: } 40 \pm 21 \\
\text { KT mattress: } 42 \pm 16 \\
\text { KL simple: } 36 \pm 16 \\
\text { KL mattress: } 53 \pm 28 \\
\text { Native labrum: } 64 \pm 22\end{array}$ & $\begin{array}{l}\text { KT simple: } 614 \pm 193 \\
\text { KT mattress: } 819 \pm 263 \\
\text { KL simple: } 696 \pm 135 \\
\text { KL mattress: } 714 \pm 178 \\
\text { Native labrum: } 688 \pm 242\end{array}$ & $\begin{array}{l}\text { Less suture slippage } \\
\text { with knotless } \\
\text { repair (11\% vs } \\
30 \%) \\
\text { Less soft tissue } \\
\text { failure with } \\
\text { mattress stitch } \\
(36 \% \text { vs } 47 \%)\end{array}$ & $\begin{array}{l}\text { No difference in } \\
\text { biomechanical } \\
\text { properties between } \\
\text { knot-tying repair, } \\
\text { knotless repair, and } \\
\text { uninjured labrum }\end{array}$ \\
\hline $\begin{array}{l}\text { Martetschläger } \\
\text { et al. }{ }^{18} \\
(2014)\end{array}$ & 18 & $\begin{array}{l}6 \text { pairs of shoulders } \\
\text { randomized to } \\
\text { knotless suture vs } \\
\text { knotless suture } \\
\text { tape repair } \\
\text { Remaining } 6 \\
\text { shoulders left } \\
\text { intact to test } \\
\text { strength of native } \\
\text { labrum }\end{array}$ & N/A & $\begin{array}{l}\text { Three knotless } \\
\text { 2.9-mm } \\
\text { BioComposite } \\
\text { PushLock } \\
\text { anchors } \\
\text { loaded with } \\
\text { No. 2 } \\
\text { FiberWire vs } \\
\text { LabralTape } \\
\text { (Arthrex) }\end{array}$ & $\begin{array}{l}\text { Suture: } 86.9 \pm 112 \\
\text { Tape: } 72.4 \pm 78 \\
\text { Native labrum: } 113 \pm 133\end{array}$ & $\begin{array}{l}\text { Suture: } 400 \pm 267 \\
\text { Tape: } 356 \pm 328 \\
\text { Native labrum: } 535 \pm 465\end{array}$ & $\begin{array}{l}\text { More glenoid } \\
\text { labrum } \\
\text { detachment in } \\
\text { repair groups } \\
\text { compared to } \\
\text { native }(66.7 \% \text { vs } \\
16.7 \% ; P=.012)\end{array}$ & $\begin{array}{l}\text { No difference in } \\
\text { biomechanical } \\
\text { properties between } \\
\text { knotless suture } \\
\text { repair, knotless tape } \\
\text { repair, and } \\
\text { uninjured labrum } \\
\text { Failure mode } \\
\text { analysis suggests } \\
\text { repaired } \\
\text { capsulolabrum } \\
\text { complex is likely } \\
\text { weaker than native } \\
\text { attachment }\end{array}$ \\
\hline $\begin{array}{l}\text { Ranawat } \\
\text { et al. }{ }^{20} \\
(2013)\end{array}$ & 16 & $\begin{array}{l}8 \text { pairs of shoulders } \\
\text { randomized to } \\
\text { knot-tying vs } \\
\text { knotless repair } \\
\text { (contralateral } \\
\text { side) }\end{array}$ & $\begin{array}{l}\text { Two knotted } \\
\text { Bio-Suture } \\
\text { Tak anchors } \\
\text { (Arthrex) }\end{array}$ & $\begin{array}{l}\text { Two Bioknotless } \\
\text { anchors } \\
\text { (Mitek) }\end{array}$ & $\begin{array}{l}\text { KT: } 19.8 \pm 9 \\
\text { KL: } 20.9 \pm 6\end{array}$ & $\begin{array}{l}\text { KT: } 96.9 \pm 95 \\
\text { KL: } 125.3 \pm 67\end{array}$ & $\begin{array}{l}\text { Both repair methods } \\
\text { fail more } \\
\text { frequently at } \\
\text { suture-tissue } \\
\text { interface than } \\
\text { anchor-bone } \\
\text { interface }\end{array}$ & $\begin{array}{l}\text { No difference in } \\
\text { biomechanical } \\
\text { properties between } \\
\text { knot-tying and } \\
\text { knotless repairs }\end{array}$ \\
\hline
\end{tabular}




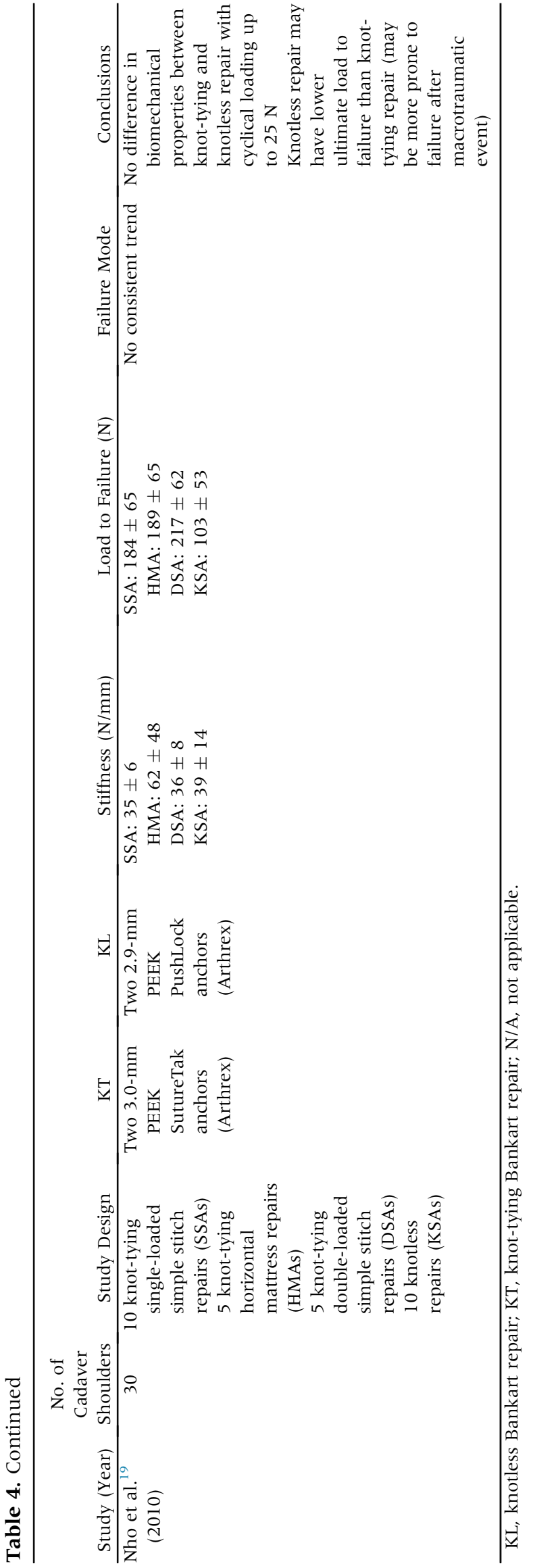

0.8 postoperatively in the knot-tying group and from 2.8 to 3.4 preoperatively and 0.9 to 1.7 postoperatively in the knotless group. All improvements in mean VAS score were statistically significant except for the knotless group in Cho et al. ${ }^{11}$ (3.2 to 1.7, P> .05). Wu et al. ${ }^{8}$ reported a higher VAS score at rest in the knotless repair group (0.7 vs $0.1, P=.021)$. Other comparative studies did not find any statistically significant difference between groups.

\section{Constant-Murley Score}

Four studies, ${ }^{10-12,17}$ involving 106 knot-tying and 130 knotless repairs, reported either a significant improvement from preoperative to postoperative or excellent postoperative Constant scores. The mean Constant score ranged from 62 to 64 preoperatively and 91 to 92 postoperatively in the knot-tying group and from 62 to 67.1 preoperatively and 86 to 94 postoperatively in the knotless group. All improvements in mean Constant score were statistically significant. No statistically significant difference between knot-tying and knotless groups was found. ${ }^{10,11}$

\section{Rowe Shoulder Score}

Nine studies, $8,9,11-17$ involving 147 knot-tying and 388 knotless repairs, reported either a significant improvement from preoperative to postoperative or excellent postoperative Rowe shoulder scores. The mean Rowe score ranged from 41 to 45 preoperatively and 92 to 93 postoperatively in the knot-tying group and from 31 to 61.6 preoperatively and 80 to 95.9 postoperatively in the knotless group. All improvements in mean Rowe score were statistically significant. No statistically significant difference between knottying and knotless groups was found..$^{8,9,11}$

\section{QuickDASH Score}

Two studies, ${ }^{8,14}$ involving 68 knot-tying and 91 knotless repairs, reported excellent postoperative QuickDASH scores. Wu et al. ${ }^{8}$ reported mean postoperative QuickDASH scores of 3.0 and 4.1 in knot-tying and knotless groups, respectively, with no difference between groups. Similarly, Alentorn-Geli et al. ${ }^{14}$ reported a mean postoperative QuickDASH score of 2.3.

\section{Return to Sport}

Four studies, $8,14,16,17$ involving 68 knot-tying and 180 knotless repairs, reported return-to-sport percentages ranging from $80 \%$ to $94 \%$ among both repair techniques. The type of sport involved included both noncontact sports such as tennis and swimming, as well as contact sports such as soccer and American football.

\section{Biomechanical Studies}

Four biomechanical studies involving a total of 94 cadaveric shoulder specimens were included. Most 
studies found no difference in biomechanical properties between knot-tying repair, knotless repair, and uninjured native labrum. In addition, the studies found that failure most frequently involved a retear at the repaired capsulolabral junction. Study design and main findings of the 4 biomechanical studies ${ }^{4,18-20}$ included in this review are summarized in Table 4 .

Lacheta et al. ${ }^{4}$ compared the biomechanical properties of knot-tying and knotless Bankart repairs using No. 2 FiberWire suture in either simple interrupted or horizontal mattress knot patterns in a 2-by-2 factorial design study involving 30 cadaveric shoulders. They found no differences in stiffness and load to failure between knot-tying repair, knotless repair, and uninjured labrum, regardless of suture pattern. Failure mode analysis revealed less suture slippage with knotless repair (11\% vs 30\%) and less soft tissue failure with the mattress suture pattern (36\% vs $47 \%$ ).

Martetschläger et al. ${ }^{18}$ compared knotless Bankart repair using No. 2 FiberWire suture vs suture tape. The authors found no differences in stiffness and load to failure between the 2 repair techniques and uninjured labrum. However, failure mode analysis showed more labral detachment in the repair groups compared with uninjured labrum $(66.7 \%$ vs $16.7 \%)$, suggesting that the repaired capsulolabrum complex is likely still weaker than native, uninjured labrum despite similar performance on biomechanical testing.

Ranawat et al. ${ }^{20}$ compared knot-tying and knotless repair in 16 cadaveric shoulders and found no differences in stiffness and load to failure. Interestingly, failure mode analysis showed that both repair methods fail more frequently at the suture-tissue interface than at the anchor-bone interface.

Finally, Nho et al. ${ }^{19}$ compared biomechanical properties and modes of failure of 3 types of knot-tying repair (single-loaded simple stitch, horizontal mattress, and double-loaded simple stitch) with knotless repair in 30 cadaveric shoulders. The authors examined both ultimate load to failure (defined as anchor pullout, suture tear, or tear at the glenolabral junction or capsulolabral tissue), as well as load to $2-\mathrm{mm}$ displacement of the repair. The repairs were stressed to failure both without cyclical loading, as well as following 100 cycles of cyclical loading from 5 to 25 Newtons, which was thought to more closely emulate shoulder biomechanics in the clinical setting. The authors found decreased load to 2-mm displacement without cyclic loading and decreased ultimate load to failure following cyclical loading in the knotless repair group. The authors concluded that knotless repair may be more prone to failure after a macrotraumatic event in the early postoperative period. The mode of failure in the knotless repair group was anchor pullout in $40 \%$ of cases and tear at the capsulolabral junction in $60 \%$ of cases.

\section{Discussion}

The principal finding from this investigation was that current level II to IV clinical evidence supports knotless arthroscopic Bankart repair as a safe and effective procedure with excellent postoperative functional outcome scores and low rates of recurrent instability and reoperation. Furthermore, current biomechanical evidence demonstrated no significant difference in stiffness and load to failure between knot-tying and knotless anchors, with the most common form of knotless anchor failure being retear at the capsulolabral junction.

The number of suture anchors required for successful arthroscopic Bankart repair has been well studied in the literature, with most studies recommending placing a minimum of $3^{21}$ or $4^{22}$ anchors to minimize risk of recurrent instability. Most studies included in our systematic review reported using 3 to 4 anchors, with the exception of Garofalo et al., ${ }^{17}$ who used 2 anchors in $40 \%(8 / 20)$ of their patients and reported a $5 \%(1 / 20)$ incidence of recurrent instability. The relatively low rate of recurrent instability across studies supports the current consensus of using a minimum of 3 to 4 suture anchors.

The knot in suture repairs is consistently the weakest point in the capsulolabral repair construct. ${ }^{23}$ To compound matters, considerable variations in knot strength exist between arthroscopic knots tied by surgeons. The literature has shown variation in knot strength to be a multifactorial phenomenon that is affected by both biomechanical factors such as suture material and knot configuration, as well as intrinsic variations in surgeon performance. Kuptniratsaikul et al. ${ }^{24}$ compared the loop security of the Weston, Chula, SMC, and Tennessee sliding knots using MagnumWire, $\mathrm{Hi}-\mathrm{Fi}$, and FiberWire sutures and found considerable variation in loop security across different knot-suture combinations. Similarly, Hassinger et al. ${ }^{25}$ found significant differences in loop security and knot security across 10 commonly used arthroscopic knots tied using the same suture material, suggesting that biomechanical factors such as knot type and suture material contribute to variation in knot strength. Furthermore, Hanypsiak et $a{ }^{26}{ }^{26}$ found not only considerable variations in knot strength between different surgeons but also inconsistency in tying 5 knots consecutively within individual surgeons. This inability to consistently tie high-quality arthroscopic knots has been identified as a drawback of knot-tying Bankart repair that would be remedied with knotless anchors.

The biomechanical strength of the conventional knot has been demonstrated to be superior to knotless technique in ex vivo studies. ${ }^{27}$ However, disadvantages of the traditional knot-tying technique include technical difficulty, high profile of the knot, and potential 
abrasion injury to the articular surface and surrounding soft tissues by the knot itself. ${ }^{28-30}$ Conversely, knotless suture anchors are technically less demanding and a reproducible means to repair labral injuries. The major drawbacks, however, in knotless technique are late disengagement of the anchor from the glenoid, as demonstrated in biomechanical studies, ${ }^{28}$ and gap formation between bone and soft tissues in cadaveric studies. ${ }^{31}$ Moreover, the knotless technique is limited to the implant device as it is typically available in a singlesuture configuration. Additional soft tissue fixation would require insertion of another anchor, making knotless anchors less amenable to bone-sparing technique and adding further monetary costs to the case.

While anchor pullout is possible with any type of anchor, failure occurs more commonly at the suturetissue interface. A biomechanical study by Ranawat et al. ${ }^{20}$ found significantly more failures at the suturetissue interface compared with pullout at the anchorbone interface (23/32 vs $9 / 32$ anchors; $P=.02)$. This is consistent with findings from our systematic review, which demonstrated a $0.7 \%(3 / 430)$ cumulative incidence for anchor pullout among knotless suture anchors. Interestingly, all 3 cases of anchor pullout occurred with all-metal anchors (Depuy Mitek) and were coincidentally diagnosed by routine postoperative plain radiographs in asymptomatic patients. Given that no cases of pullout occurred with biocomposite anchors, there exists the possibility that this complication may be associated with anchor design. The authors also postulated inadequate depth of insertion and failure to stress the anchor to ensure secure fixation after insertion as possible causes of pullout. All 3 patients underwent arthroscopic removal of the loose anchor and had intraoperative finding of humeral head cartilage damage from the backed-out anchor. All other patients who underwent revision surgery for recurrent instability had retear of the repaired capsulolabral junction with no evidence of anchor damage or pullout. Together, these findings suggest that anchor pullout is a serious complication associated with a high incidence of glenohumeral chondrosis but is fortunately very rare with modern biocomposite anchor designs.

Another proposed advantage of knotless suture anchors is potential cost-savings secondary to shorter operative time. While no study to date on knotless Bankart repair had examined operative time and overall surgical cost, this topic is beginning to be explored in the rotator cuff literature. A 2019 retrospective cohort study by Burns et al. ${ }^{32}$ evaluated the surgical cost and clinical outcomes associated with 35 knot-tying and 54 knotless double-row arthroscopic rotator cuff repairs. The authors found that despite having higher implant costs ( $\$ 2127$ vs $\$ 1520 ; P<.01)$, the knotless group had significantly lower overall surgical cost ( $\$ 3788$ vs $\$ 4263 ; P<.01$ ) due to significantly shorter operative time ( 43.5 vs 80 minutes; $P<.001$ ). No statistically significant differences in functional outcome were found between groups, as evaluated by the VAS, Simple Shoulder Test, American Shoulder and Elbow Surgeons, and University of California at Los Angeles scores. Shorter operative time results in not only significant cost-savings for the health care system but may also decrease the side effects associated with general anaesthesia such as nausea, vomiting, and somnolence. Given the promising early results from the rotator cuff literature, this issue warrants further exploration in future Bankart repair studies.

The strengths of this study include its comprehensive literature review, rigorous methodology, and the fact that it addresses a clinical question that has demonstrated clinical equipoise in the literature to date. At present, there is insufficient evidence to support the use of one type of anchor over another. Both traditional and knotless suture anchors have demonstrated excellent results in the current body of literature. Further scientific investigation is warranted to determine if superiority of one technique exists. Given that the differences in outcomes between traditional and knotless groups across studies were small, a power analysis would be useful to determine how many subjects would be needed to appropriately power such a trial and minimize the risk of type II error. Future research addressing the issue of traditional vs knotless arthroscopic sutures should use a randomized study design to evenly distribute random variation between treatment groups, and it should include a large number of study subjects from a diverse, generalizable population. Appropriate subgroup analysis would be necessary to study patients with varying demographic profiles and activity levels to determine whether or not long-term outcome is similar between traditional and knotless groups.

\section{Limitations}

This systematic review has several limitations. First, the studies included in this review are of low-quality evidence. There were no level I randomized studies and only 2 level II prospective cohort studies. As a result, data pooling and quantitative meta-analysis were not feasible without significantly increasing selection bias. Second, the scarcity of studies comparing knot-tying and knotless repair, as well as the small sample size and nonrandomized nature of available comparative studies, limited our ability to draw conclusions regarding the superiority of one technique over the other. Third, the included studies had multiple sources of heterogeneity, including pathology, patient demographics, surgical technique, implants used, and postoperative protocols. For instance, Kocaoglu et al. ${ }^{9}$ used both screw-in and malleted anchors, while other studies only used a screw-in insertion technique. 
Similarly, earlier studies used metal anchors while more recent studies primarily used bioabsorbable PEEK anchors. Furthermore, there were variations across studies in the patient-reported outcomes used to evaluate postoperative function. The resulting heterogeneity was estimated to be moderate (37\%-42\%) using the $I^{2}$ statistic. While exploration of the sources of heterogeneity showed similar findings across all studies without any obvious confounding variables, the considerable heterogeneity nonetheless precluded data pooling and formal meta-analysis. Last, we were unable to explore the financial implications of knot-tying vs knotless Bankart repair as none of the included studies reported operative time.

\section{Conclusions}

Current level II to IV clinical and biomechanical evidence supports knotless arthroscopic Bankart repair as an effective procedure with excellent functional outcomes and low rates of complication and reoperation.

\section{References}

1. Ide J, Maeda S, Takagi K. Arthroscopic Bankart repair using suture anchors in athletes: Patient selection and postoperative sports activity. Am J Sports Med 2004;32: 1899-1905.

2. Wang C, Ghalambor N, Zarins B, Warner JJ. Arthroscopic versus open Bankart repair: Analysis of patient subjective outcome and cost. Arthroscopy 2005;21:1219-1222.

3. Cooke SJ, Starks I, Kathuria V. The results of arthroscopic anterior stabilisation of the shoulder using the bioknotless anchor system. Sports Med Arthrosc Rehabil Ther Technol 2009;1:2.

4. Lacheta L, Brady A, Rosenberg SI, et al. Biomechanical evaluation of knotless and knotted all-suture anchor repair constructs in 4 Bankart repair configurations. Arthroscopy 2020;36:1523-1532.

5. Moher D, Liberati A, Tetzlaff J, Altman DG. Preferred reporting items for systematic reviews and meta-analyses: The PRISMA statement. PLoS Med 2009;6:e1000097.

6. Slim K, Nini E, Forestier D, Kwiatkowski F, Panis Y, Chipponi J. Methodological Index for Non-Randomized Studies (MINORS): Development and validation of a new instrument. ANZ J Surg 2003;73:712-716.

7. Higgins JP, Thompson SG. Quantifying heterogeneity in a meta-analysis. Stat Med 2002;21:1539-1558.

8. Wu IT, Desai VS, Mangold DR, et al. Comparable clinical outcomes using knotless and knot-tying anchors for arthroscopic capsulolabral repair in recurrent anterior glenohumeral instability at mean 5-year follow-up [published online May 18, 2020]. Knee Surg Sports Traumatol Arthrosc.

9. Kocaoglu B, Guven O, Nalbantoglu U, Aydin N, Haklar U. No difference between knotless sutures and suture anchors in arthroscopic repair of Bankart lesions in collision athletes. Knee Surg Sports Traumatol Arthrosc 2009;17: 844-849.
10. Ng DZ, Kumar VP. Arthroscopic Bankart repair using knot-tying versus knotless suture anchors: Is there a difference? Arthroscopy 2014;30:422-427.

11. Cho NS, Lubis AM, Ha JH, Rhee YG. Clinical results of arthroscopic Bankart repair with knot-tying and knotless suture anchors. Arthroscopy 2006;22:1276-1282.

12. Stein T, Linke RD, Buckup J, et al. Shoulder sport-specific impairments after arthroscopic Bankart repair: a prospective longitudinal assessment. Am J Sports Med 201 1;39: 2404-2414.

13. Thal R, Nofziger M, Bridges M, Kim JJ. Arthroscopic Bankart repair using knotless or bioknotless suture anchors: 2- to 7-year results. Arthroscopy 2007;23:367-375.

14. Alentorn-Geli E, Alvarez-Diaz P, Doblas J, et al. Return to sports after arthroscopic capsulolabral repair using knotless suture anchors for anterior shoulder instability in soccer players: Minimum 5-year follow-up study. Knee Surg Sports Traumatol Arthrosc 2016;24:440-446.

15. Hayashida K, Yoneda M, Mizuno N, Fukushima S, Nakagawa S. Arthroscopic Bankart repair with knotless suture anchor for traumatic anterior shoulder instability: Results of short-term follow-up. Arthroscopy 2006;22: 620-626.

16. Park JY, Lhee SH, Park HK, Jeon SH, Oh JH. Perianchor radiolucency after knotless anchor repair for shoulder instability: Correlation with clinical results of 69 cases. Am J Sports Med 2009;37:360-370.

17. Garofalo R, Mocci A, Moretti B, et al. Arthroscopic treatment of anterior shoulder instability using knotless suture anchors. Arthroscopy 2005;21:1283-1289.

18. Martetschläger F, Michalski MP, Jansson KS, Wijdicks CA, Millett PJ. Biomechanical evaluation of knotless anterior and posterior Bankart repairs. Knee Surg Sports Traumatol Arthrosc 2014;22:2228-2236.

19. Nho SJ, Frank RM, Van Thiel GS, et al. A biomechanical analysis of anterior Bankart repair using suture anchors. Am J Sports Med 2010;38:1405-1412.

20. Ranawat AS, Golish SR, Miller MD, et al. Modes of failure of knotted and knotless suture anchors in an arthroscopic Bankart repair model with the capsulolabral tissues intact. Am J Orthop (Belle Mead NJ) 2011;40:134-138.

21. Kim SH, Ha KI, Kim SH. Bankart repair in traumatic anterior shoulder instability: Open versus arthroscopic technique. Arthroscopy 2002;18:755-763.

22. Boileau P, Villalba M, Héry JY, Balg F, Ahrens P, Neyton L. Risk factors for recurrence of shoulder instability after arthroscopic Bankart repair. J Bone Joint Surg Am 2006;88:1755-1763.

23. Leedle BP, Miller MD. Pullout strength of knotless suture anchors. Arthroscopy 2005:21:81-85.

24. Kuptniratsaikul S, Weerawit P, Kongrukgreatiyos K, Promsang T. Biomechanical comparison of four sliding knots and three high-strength sutures: Loop security is much different between each combination. J Orthop Res 2016;34:1804-1807.

25. Hassinger SM, Wongworawat MD, Hechanova JW. Biomechanical characteristics of 10 arthroscopic knots. Arthroscopy 2006;22:827-832.

26. Hanypsiak BT, DeLong JM, Simmons L, Lowe W, Burkhart S. Knot strength varies widely among expert arthroscopists. Am J Sports Med 2014;42:1978-1984. 
27. Sileo MJ, Lee SJ, Kremenic IJ, et al. Biomechanical comparison of a knotless suture anchor with standard suture anchor in the repair of type II SLAP tears. Arthroscopy 2009:25:348-354.

28. Hovelius L, Thorling J, Fredin H. Recurrent anterior dislocation of the shoulder: Results after the Bankart and PuttiPlatt operations. J Bone Joint Surg Am 1979;61:566-569.

29. Thal R. Knotless suture anchor: Arthroscopic Bankart repair without tying knots. Clin Orthop Relat Res 2001;390: 42-51.
30. Zumstein M, Jacob HA, Schneeberger AG. In vitro comparison of standard and knotless metal suture anchors. Arthroscopy 2004;20:517-520.

31. Rowe CR, Patel D, Southmayd WW. The Bankart procedure: A long-term end-result study. J Bone Joint Surg Am 1978;60:1-16.

32. Burns KA, Robbins L, LeMarr AR, Childress AL, Morton DJ, Wilson ML. Rotator cuff repair with knotless technique is quicker and more cost-effective than knotted technique. Arthrosc Sports Med Rehabil 2019;1:e123-e130. 